\title{
Modélisation expérimentale et numérique d'un tourbillon de vidange avec injection en régime super-critique
}

\author{
Gaspard FOURESTIER ${ }^{1,2,3}$, Timothée SANTAGOSTINI ${ }^{1}$, Marc LE BOULLUEC ${ }^{2}$, Philippe MAGALDI ${ }^{1}$, \\ Yves-Marie SCOLAN ${ }^{3}$
}

${ }^{1}$ GEPS Techno - e-mail: gaspard.fourestier@geps-techno.com
${ }^{2}$ Ifremer
${ }^{3}$ ENSTA Bretagne

RÉSUMÉ. - Un type d'usine hydroélectrique pour faible hauteur de chute est étudié. Il se base sur la formation d'un tourbillon de vidange au sein duquel est plongée une turbine. Dans un premier temps le tourbillon de vidange est étudié sans turbine. Une campagne expérimentale permettant l'étude de ce tourbillon de vidange est conduite. Lors de cette campagne, les hauteurs d'eau et les vitesses dans le tourbillon sont mesurées. Ces dernières sont déterminées en utilisant un système de vélocimétrie laser (Laser Doppler Velocimetry, LDV). Un modèle numérique du tourbillon est réalisé avec le solveur interFoam du logiciel OpenFOAM 2.2.2, avec une approche diphasique. Les données expérimentales sont comparées qualitativement et quantitativement aux données numériques. Un calcul est réalisé en laminaire et les modèles de turbulence k- $\varepsilon$ RNG et k- $\omega$ SST sont testés. Globalement le calcul laminaire et le modèle k- $\omega$ SST représente correctement le phénomène physique observé. Les vitesses proches de la surface libre sont moins bien estimées.

Mots-clés : tourbillon de vidange, interFoam, vélocimétrie laser

\section{Experimental and numerical simulation of a supercritical bathtub vortex with an injection}

\begin{abstract}
One type of low head hydropower plant is studied. This solution, based on a bathtub vortex, is called the gravitational vortex hydropower. It consists in a turbine placed at the center of the vortex which harvests the vortex kinetic energy. As a first step, the bathtub vortex is studied without the turbine, in the supercritical flow regime. An experimental campaign is carried out to study this bathtub vortex. Water heights are measured using acoustic sensors in seven points in the tank. A laser Doppler velocimetry device is deployed to measure the velocities in three planes in the water flow. Simulations are performed using OpenFOAM 2.2.2 with a two phase approach using the volume of fluid method (solver interFoam). First simulations are performed without any modelling of turbulent phenomena (laminar model), afterwards the k- $\varepsilon$ RNG model and the k- $\omega$ SST model are tested. The experimental data are compared qualitatively and quantitatively to the values extracted from the numerical model. The laminar and k- $\omega$ SST turbulence model calculate accurately the water heights in the tank. The water heights predicted by the k- $\varepsilon$ RNG turbulence model are lower than the measured ones. Generally, velocity profiles calculated by laminar and k- $\omega$ SST turbulence model are close to the experimental. In the vicinity of the free surface differences are higher. Radial and axial velocities calculated by the k- $\omega$ SST turbulence model are closer to the experimental velocities than those computed with the laminar model.
\end{abstract}

Key-words: bathtub vortex, interFoam, laser Doppler velocimetry

\section{INTRODUCTION}

Afin de répondre à la demande croissante en énergie sans émettre davantage de gaz à effet de serre, il est nécessaire de trouver de nouvelles sources d'énergie. Avec 16.3\% de l'électricité produite dans le monde en 2013 [International Energy Agency, 2015], l'énergie hydraulique fait déjà partie de la solution. Cependant, la plupart des sites qui ont les caractéristiques permettant la construction des usines hydroélectriques traditionnelles (hauteur de chutes supérieure à $3 \mathrm{~m}$ [Power et al., 2016]) sont déjà exploités. De petites usines électriques sont donc maintenant imaginées. Ces solutions ont l'avantage d'avoir de faibles impacts environnementaux et elles peuvent être installées dans des régions avec de faibles demandes en électricité. Une de ces solutions, parfois appelée Gravitational Vortex Hydropower (GVHP) en anglais, est une usine hydroélectrique basée sur un tourbillon de vidange. Certaines entreprises développent cette solution [Zotlöterer, 2004], et des installations jusqu'à $18 \mathrm{~kW}$ sont déjà utilisées. Pour extraire le maximum d'énergie d'un tourbillon de vidange, il est tout d'abord important de bien comprendre ce phénomène. Le but de cet article est donc de participer à la compréhension de celui-ci. Contrairement à la plupart des études sur le sujet dont le but final est d'empêcher sa formation, on cherche ici la formation du tourbillon le plus intense possible. La configuration étudiée consiste en un réservoir circulaire équipé en partie haute d'une admission d'eau qui détermine le débit dès lors que le niveau d'eau moyen interne demeure constant, et en partie centrale basse d'un diaphragme circulaire qui permet la sortie d'eau et au-dessus duquel se met en place un siphon ou tourbillon de vidange.

\section{I.1. Le tourbillon de vidange : les modèles analytiques}

Des études mettent en évidence différents régimes d'écoulement pour le tourbillon de vidange. Plusieurs classifications 
sont proposées [Daggett and Keulegan, 1974; Odgaard, 1986; Stepanyants and Yeoh, 2008; Cristofano et al., 2014a]. Celle utilisée ici est constituée de 3 niveaux (Figure 1). Le premier niveau est le régime sub-critique, il correspond au cas où il n'y a pas d'entraînement d'air à travers le diaphragme. Le second niveau est appelé régime critique, il est observé lorsque la pointe du vortex atteint exactement le diaphragme (qui est l'ouverture au-dessus de laquelle se forme le tourbillon). Le troisième et dernier régime, lorsqu'il y a un entrânement d'air à travers le diaphragme, est le régime supercritique. Dans le cas de la création d'énergie, le tourbillon considéré est toujours supercritique.

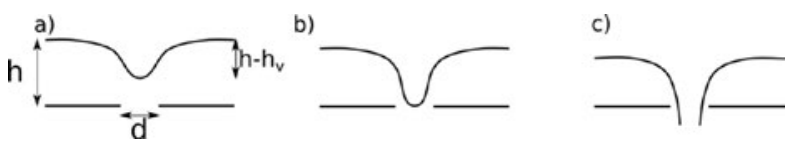

Figure 1 : Classification des tourbillons de vidange. a) vortex subcritique. b) vortex critique. c) vortex supercritique. $d$ : diamètre du diaphragme, $h:$ hauteur d'eau, $h-h v: p r o-$ fondeur du tourbillon

Plusieurs modèles analytiques du profil de vitesse dans le tourbillon sont proposés dans la littérature [Rankine, 1872; Odgaard, 1986; Hite Jr. and Mih, 1995; Chen et al., 2007]. Ces modèles font l'hypothèse d'un écoulement stationnaire, axisymétrique dans un domaine infini. Des hypothèses additionnelles sont faites sur le profil de vitesse selon le modèle proposé (par exemple, la vitesse tangentielle est supposée proportionnelle à la circulation du tourbillon et inversement proportionnelle au rayon, ou la vitesse radiale est considéré proportionnelle au rayon). Tous ces modèles sont fondés sur l'hypothèse d'une faible profondeur du vortex par rapport à la profondeur d'eau [Stepanyants and Yeoh, 2008]. Cette hypothèse n'est pas vérifiée pour un vortex supercritique.
Lundgren [1985] a proposé un modèle numérique pour les tourbillons de vidange stationnaires dans un récipient tournant. Celui-ci a été amélioré par Andersen et al [2006] et appliqué par Stepanyants and Yeoh [2008] au cas du tourbillon de vidange dans un récipient fixe. L'intérêt de ce modèle est qu'il reste valide dans le cas de tourbillons critiques ou supercritiques.

Dans tous ces modèles, la circulation est un des paramètres d'entrée. Dans des applications avec des géométries complexes, cette grandeur peut être difficile à évaluer précisément a priori. Ceci limite l'utilisation pratique de ces modèles mais permet de comprendre l'effet des différents paramètres sur le phénomène physique étudié.

En réalisant une analyse dimensionnelle, différents auteurs montrent que six paramètres adimensionnels sont nécessaires pour représenter le tourbillon de vidange [Daggett and Keulegan, 1974; Anwar et al., 1978]. Parmi ceux-ci les nombres de Reynolds, Froude et Weber sont utilisés. Cependant les définitions de ces nombres varient d'un auteur à l'autre. Le Tableau 1 résume les différentes définitions utilisées. Les deux paramètres suivants sont des paramètres géométriques : $\mathrm{D} / \mathrm{d}$ et $\mathrm{H} / \mathrm{d}$ où $\mathrm{d}$ est le diamètre $\mathrm{du}$ diaphragme, $\mathrm{D}$ est le diamètre du contenant et $\mathrm{H}$ est la hauteur d'eau loin du vortex. Le dernier paramètre est un rapport entre les vitesses tangentielles et radiales, il est évalué par Dagget et Keulegan [1974] avec : $(\Gamma \mathrm{d}) / \mathrm{Q}$ où $\Gamma$ est la circulation du tourbillon et $\mathrm{Q}$ est le débit dans l'installation.

Recoquillon [2013] présente une étude complète du tourbillon de vidange. Les mouvements du vortex sont étudiés et des profils de vitesse sont mesurés par PIV (Particle Image Velocimetry) dans des plans verticaux et horizontaux.

Cristofano et al. [2016] mesurent par PIV les vitesses dans le tourbillon dans trois plans horizontaux pour différents régimes du vortex. Ils étudient les mouvements $\mathrm{du}$ centre du vortex et des profils de vitesses radiales et tangentielles pour les différents régimes d'écoulement du vortex et dans des plans à différentes hauteurs. Ils comparent les mesures obtenues au modèle analytique de Rankine[1872], et constatent que le modèle de Rankine donne une bonne

Tableau 1: Résumé des différentes définitions données par plusieurs auteurs des différents nombres sans dimensions utilisés.

Re est le nombre de Reynolds, Fr est le nombre de Froude, We est le nombre de Weber, $Q$ est le débit, $d$ est le diamètre du diaphragme, $v$ est la viscosité cinématique, g est l'accélération de la pesanteur, $\sigma$ est la tension de surface du liquide dans le gaz, $\rho$ est la masse volumique du liquide, $h$ est le niveau d'eau au-dessus du diaphragme, $h$ 'est le niveau d'eau au-dessus du fond du diaphragme ( $h$ ' $=h+e$, où e est l'épaisseur du diaphragme), $U$ est la vitesse moyenne en sortie du bassin.

\begin{tabular}{|c|c|c|c|}
\hline & $\operatorname{Re}$ & $\mathrm{Fr}$ & We \\
\hline Daggett et al.[1974] & $\frac{Q}{\frac{d}{2} v}$ & $\frac{Q}{\frac{\pi d^{2}}{4} \sqrt{g h^{\prime}}}$ & $\frac{Q}{2 \pi \frac{d}{2}} \sqrt{\frac{\rho}{\sigma \frac{d}{2}}}$ \\
\hline Anwar et al. [1978] & $\frac{Q}{v h}$ & $\frac{Q}{\frac{\pi d^{2}}{4} \sqrt{2 g h}}$ & $\frac{\rho Q^{2} h}{\left(\frac{\pi d^{2}}{4}\right)^{2} \sigma}$ \\
\hline Cristofano et al.[2014a] & $\frac{U d}{v}$ & $\frac{U}{\sqrt{g h}}$ & $\frac{\rho U^{2} d}{\sigma}$ \\
\hline $\begin{array}{c}\text { Notations utilisées dans cet } \\
\text { article }\end{array}$ & $\frac{Q}{d v}$ & $\frac{Q}{\frac{\pi d^{2}}{4} \sqrt{g h^{\prime}}}$ & $\frac{Q}{2 \pi \frac{d}{2}} \sqrt{\frac{\rho}{\sigma \frac{d}{2}}}$ \\
\hline
\end{tabular}


représentation du profil de vitesse tangentielle pour les plans les plus proches de l'interface. Li et al. [2008] utilisent également la PIV pour déterminer les profils de vitesse tangentielle dans un bassin rectangulaire. Ils comparent les profils de vitesse obtenus pour différents régimes d'écoulement. Ils observent que les vitesses tangentielles sont les plus importantes lorsqu'il y a entraînement d'air. Par ailleurs, ils font des comparaisons qualitatives avec leur modèle numérique qui reproduit correctement le phénomène observé.

\section{I.2. Le tourbillon de vidange: modèles numériques}

Pour modéliser le tourbillon de vidange, certains auteurs utilisent des modèles monophasiques [Li et al., 2008; Bøhling et al., 2010] et d'autres utilisent des modèles diphasiques [Chen et al., 2012; Recoquillon, 2013]. Chen et al. [2012] conduisent des simulations diphasiques du vortex avec quatre injections symétriques dans un bassin carré en implémentant la méthode VOF (Volume of Fluid). Ils comparent deux modèles de turbulence différents : le modèle k- $\varepsilon$ standard [Chassaing, 2000] et le modèle RNG k- $\varepsilon$ [Speziale and Thangam, 1992]. Ils montrent que le second modèle est préférable car il permet la formation du vortex alors que le premier ne permet pas le creusement de la surface libre observé dans les expériences. Recoquillon [2013] réalise des simulations du tourbillon en utilisant le logiciel OpenFOAM. Il utilise un solveur appelé interFOAM qui implémente la méthode VOF pour déterminer l'interface entre les deux phases [Deshpande et al., 2012]. Il compare ces résultats avec les expériences qu'il a réalisées et montre des difficultés à représenter correctement le niveau d'eau observé lors des expériences. Le niveau d'eau calculé est toujours sous-estimé. Les profils de vitesse calculés sont en accord avec les mesures loin de l'interface mais ne sont pas satisfaisants proche de l'interface et du cœur du tourbillon, en particulier pour les vitesses radiales et tangentielles.

Cristofano et al. [2014b; 2015] étudient l'influence du modèle de turbulence sur le profil de l'interface pour des tourbillons de vidange sub-critiques. Ils comparent les résultats de simulations avec trois modèles de turbulence différents : le modèle laminaire (sans modèle de turbulence particulier), le modèle k- $\omega$ standard couplé avec un modèle SAS (Scale Adaptive Simulation) [Cristofano et al., 2014b] et le modèle SST (Shear-Stress Transport) k- $\omega$ couplé avec le modèle SAS [Cristofano et al., 2014b]. Ils comparent ces simulations aux données expérimentales qu'ils ont obtenues et ils mettent en évidence que le meilleur modèle est le modèle SST k- $\omega$.

Diverses études sur les tourbillons de vidange existent déjà. Mais l'étude des tourbillons de vidange supercritiques est plus rare, en particulier en présence d'une injection. Nous présentons ici une campagne expérimentale sur ce type de tourbillon ainsi qu'un modèle numérique de celui-ci. Les données expérimentales et numériques sont enfin comparées.

\section{DISPOSITIF EXPÉRIMENTAL :}

Le but est de représenter un tourbillon de vidange supercritique, confiné et avec une injection. Le système expérimental est décrit sur la Figure 2 [Fourestier, 2017]. L'eau s'écoule du bassin d'étude pour rejoindre le bassin de récupération à travers le diaphragme situé au centre du bassin d'étude. Le bassin d'étude est un récipient presque cylindrique qui a un diamètre de $390 \mathrm{~mm}$. Une pompe est utilisée pour monter l'eau du bassin de récupération vers le canal d'amenée et maintenir un écoulement quasi-stationnaire dans le bassin d'étude.

Les trois ratios entre le diamètre du diaphragme (d) et le diamètre du récipient (D) testés sont $0.51,0.33$ et 0.26 . Pendant la campagne expérimentale trois pompes différentes sont utilisées. Elles sont appelées GP, MP et PP, et se distinguent par leur plage de débit. Une vanne permet de contrôler le débit des pompes GP et MP.

Les hauteurs d'eau dans le bassin d'étude et dans le canal d'amenée sont mesurées avec des capteurs acoustiques en sept positions montrées sur la Figure 3. Le débit de la pompe et la vitesse de l'eau est mesurée pour cinq configurations différentes. Chaque configuration est définie par une valeur unique du doublet de paramètres : diamètre du diaphragme, débit de la pompe. Ces configurations sont listées dans le Tableau 2.

Les trois composantes de la vitesse sont mesurées par des systèmes « Laser Doppler Velocimetry » (LDV) fournis par Dantec Dynamics et appelés FlowExplorer. Deux FlowExplorer

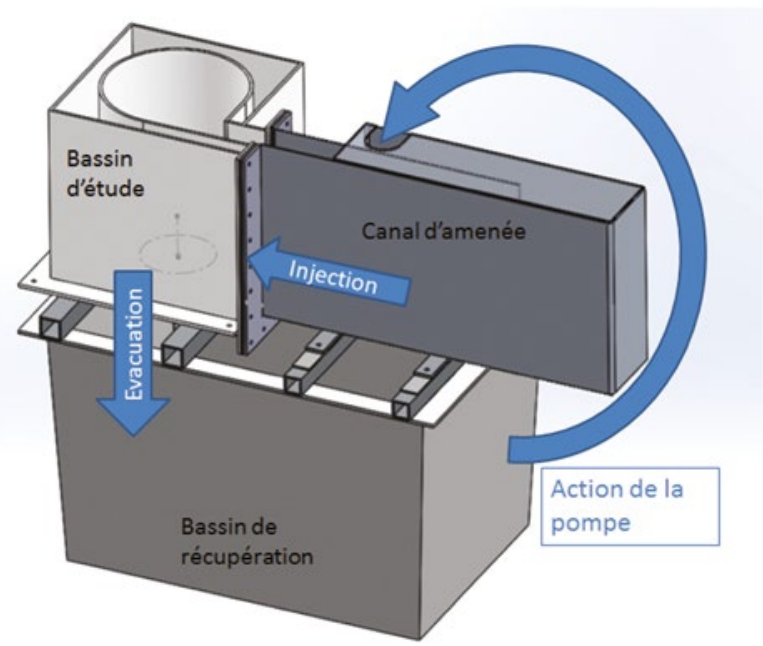

Figure 2 : Schéma de principe du montage expérimental. Les flèches bleues représentent le sens de circulation de l'eau. Le tourbillon de vidange est observé dans le bassin d'étude. Le mouvement d'eau en circuit fermé est maintenu par l'intermédiaire d'une pompe.

Tableau 2 : Tableau des configurations étudiées. Le nombre de Reynolds (Re) et le nombre de Froude (Fr) sont évalués avec les formulations qui sont présentées dans le Tableau 1.

\begin{tabular}{|c|c|c|c|c|c|c|}
\hline Configuration & $\mathrm{d}(\mathrm{mm})$ & Pompe & $\mathrm{h}(\mathrm{cm})$ & $\mathrm{Q}(1 / \mathrm{s})$ & $\operatorname{Re}$ & $\mathrm{Fr}$ \\
\hline 1 & 130 & $\mathrm{pp}$ & 16.4 & 3.5 & 27385 & 0.22 \\
\hline 2 & 100 & MP2 & 28.6 & 5.5 & 55239 & 0.42 \\
\hline 3 & 200 & MP8 & 28.7 & 14.0 & 70237 & 0.28 \\
\hline 4 & 130 & MP3 & 32.9 & 9.4 & 72423 & 0.40 \\
\hline 5 & 130 & GP2 & 26.3 & 7.2 & 55371 & 0.34 \\
\hline
\end{tabular}




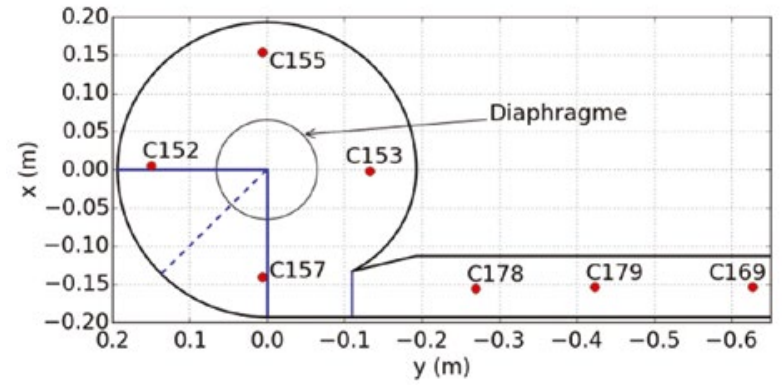

Figure 3: Schéma de la position des sondes à ultrason et des plans de mesure des vitesses. Les noms des capteurs à ultrason sont composés d'une lettre (" $C »)$ et de trois nombres (par exemple " $178 »)$. Les plans où la vitesse est mesurée sont représentés en bleu.

sont utilisés : un FlowExplorer bi-composante et un FlowExplorer mono-composante. Ces trois systèmes mesurent les trois composantes de la vitesse en un point de l'espace. Ils sont donc déplacés pour couvrir les plans du bassin d'étude présenté à la Figure 3. Le déplacement des FlowExplorer est pris en charge par un système de déplacement automatique contrôlé par l'ordinateur collectant les données.

\section{III. ÉTUDE NUMÉRIQUE}

Le modèle numérique du tourbillon de vidange est réalisé en utilisant le solveur interFoam du logiciel OpenFOAM [OpenFOAM Foundation, 2013]. Ce solveur résout les équations de Navier-Stokes pour des écoulements diphasiques en utilisant la méthode VOF [Deshpande et al., 2012] pour déterminer la position de l'interface. Il suppose que les fluides sont incompressibles et non miscibles. Dans un premier temps, les phénomènes turbulents ne sont pas représentés dans ce modèle (modèle laminaire). En se basant sur la bibliographie présentée, les modèles de turbulence $\mathrm{k}-\varepsilon$ RNG et k- $\omega$ SST sont testés dans un second temps. Le pas de temps est automatiquement ajusté par OpenFOAM afin de vérifier la condition de Courant-Friedrichs-Lewy (CFL), avec un nombre de Courant maximum imposé à 0.5.

La simulation débute avec un bassin partiellement rempli d'eau au repos. La hauteur d'eau initiale est fixée a priori. A l'injection, une vitesse constante est imposée. Sa valeur est déterminée à partir du débit mesuré lors des expériences. La vitesse sur les murs est nulle. A la sortie et sur la condition aux limites ouverte à l'atmosphère (paroi supérieure du maillage) est imposée une condition permettant la sortie des deux fluides du domaine modélisé, ou une entrée d'air si nécessaire. Ainsi, lorsque la vitesse est orientée vers l'extérieur, une condition de Neumann est appliquée et lorsque la vitesse est orientée vers l'intérieur du domaine, une condition de Dirichlet est appliquée (la valeur de la vitesse perpendiculairement à la paroi est estimée à partir du flux du pas de temps précédent).

Le maillage est, initialement, un maillage structuré par bloc, mais des zones de raffinement sont ajoutées près des murs et dans la zone de l'interface (Figure 4) rendant le maillage non structuré. Des maillages (décrits dans le Tableau 3) avec des tailles de mailles décroissantes sont comparés. Ces trois maillages ont des $\mathrm{y}+$ moyen de l'ordre de 9 ( $y+$ est la distance normalisée entre le centre de la première maille et la paroi [Recoquillon, 2013]). Les profils verticaux de vitesses tangentielles et de fraction volumique dans le bassin d'étude sont comparés dans la Figure 5. Lorsque la fraction volumique vaut 1 le fluide considéré est

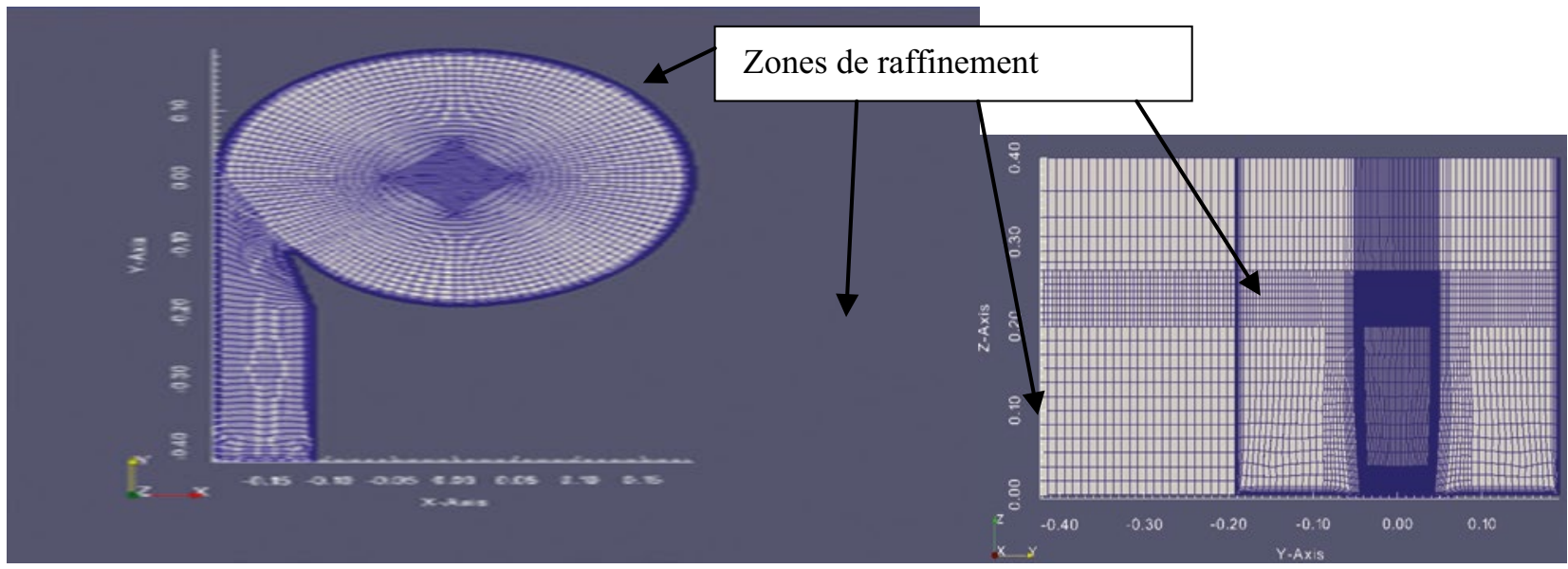

Figure 4 : Maillage utilisé pour la 5ième configuration (cas avec des mailles de taille intermédiaire)

Tableau 3 : Présentation des différents maillages utilisés. Le temps de calcul du maillage le plus fin est de $419 \mathrm{~h}$ pour $60 \mathrm{~s}$ de temps physique, il est ramené aux valeurs indiquées dans le tableau pour $25 \mathrm{~s}$ de temps physique en supposant que le temps de calcul est proportionnel à la durée simulée.

\begin{tabular}{|l|c|c|c|c|}
\hline \multicolumn{1}{|c|}{ Nom du cas } & Nombre de cellules & $\begin{array}{c}\text { Taille des mailles près } \\
\text { du diaphragme }(\mathrm{mm})\end{array}$ & $\begin{array}{c}\text { Nombre de maille sur } \\
\text { le pourtour du cylindre }\end{array}$ & $\begin{array}{c}\text { Temps de calcul } \\
\text { Pour 25s (h) }\end{array}$ \\
\hline maillage grossier & 315363 & 2.1 & 96 & 53 \\
\hline maillage intermédiaire & 425011 & 1.6 & 128 & 77 \\
\hline maillage fin & 998198 & 1.3 & 160 & 175 \\
\hline
\end{tabular}




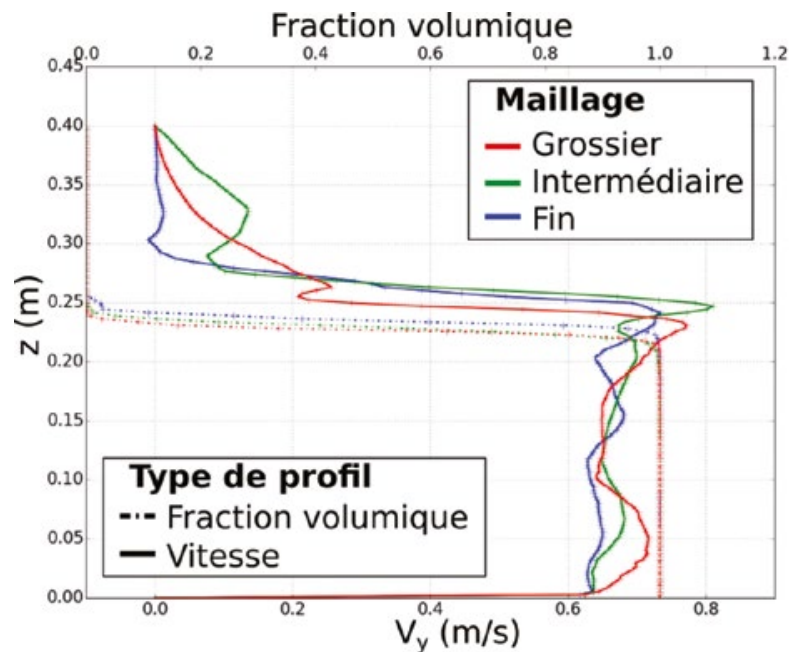

Figure 5 : Profils verticaux de vitesse tangentielle et de fraction volumique, comparaison pour trois tailles de maille différentes. Le profil est réalisé au point de coordonnées horizontales suivant : $(-0.140,0.006)$.

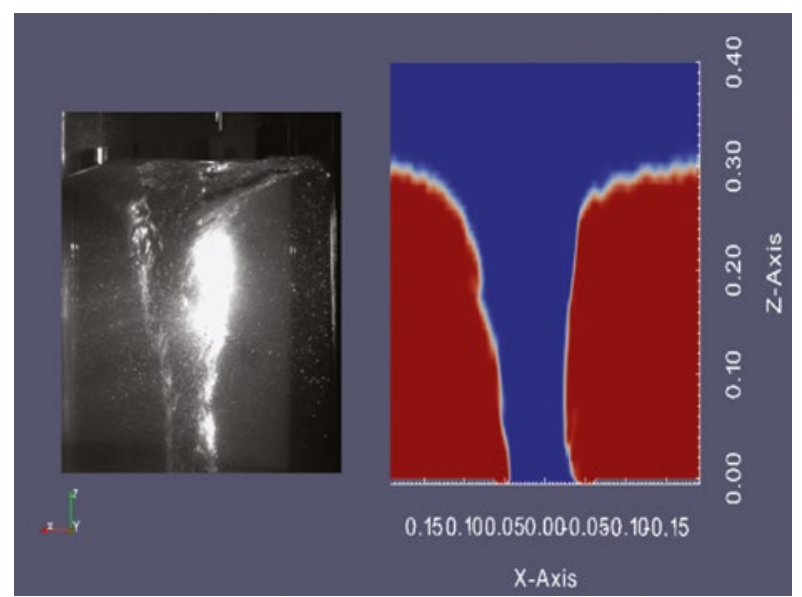

Figure 6 : Comparaison qualitative entre une photo du vortex observé lors des expériences correspondant à la 5ième configuration (a) et le vortex issu du modèle numérique correspondant (b) a)

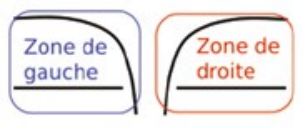

b)

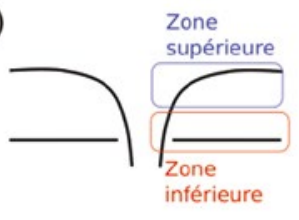

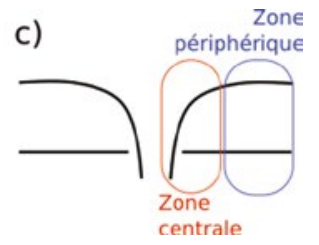

Figure 7 : Description des différentes zones du vortex

l'eau et lorsqu'elle est nulle le fluide considéré est l'air. Les profils présentés sont des profils de valeurs instantanées pour le temps $\mathrm{t}=25 \mathrm{~s}$. À cet instant l'écoulement est stationnaire en moyenne (il subit des variations autour d'un écoulement moyen). On observe que le maillage le plus fin correspond au cas où l'interface est la plus nette (gradient de fraction volumique le plus important) et est donc la mieux définie. Pour des raisons de précision et de temps de calcul acceptables, le maillage de taille intermédiaire est utilisé ensuite. Les simulations réalisées avec les modèles de turbulences k- $\varepsilon$ RNG et k- $\omega$ SST utilisent le maillage intermédiaire présenté ici. Cependant, pour ces deux cas le maillage proche des parois est modifié pour atteindre un $\mathrm{y}+$ moyen de 90 pour le modèle $\mathrm{k}-\varepsilon$ RNG et de 57 pour le modèle $\mathrm{k}-\omega$ SST. Dans les deux cas, des lois de paroi sont utilisées.

\section{DISCUSSION}

\section{IV.1. Comparaison entre le calcul laminaire et les résultats expérimentaux}

Dans un premier temps les données expérimentales sont comparées uniquement avec les résultats du calcul laminaire. La Figure 6 présente une comparaison entre les interfaces du modèle numérique et celles observées lors des expériences. Dans cette figure, la hauteur d'eau près des parois et le profil $\mathrm{du}$ vortex sont similaires dans les deux cas. Pour l'analyse de la Figure 6a) plusieurs zones de l'interface sont distinguées : i) les zones gauche et droite, ii) la partie centrale et la partie périphérique, iii) les parties supérieures et inférieures. Ces zones sont présentées à la Figure 7 pour améliorer la compréhension. Sur la Figure 6a), on observe une différence de comportement entre la zone de droite, où l'interface est presque verticale, et la zone de gauche où l'interface forme un angle plus grand avec la verticale en particulier dans la zone supérieure. Cette différence est expliquée par la présence de l'injection qui provoque un défaut d'axisymétrie dans le domaine. Cette asymétrie explique aussi le décentrage du tourbillon par rapport à la géométrie, en particulier dans la zone supérieure. Dans la zone inférieure, le tourbillon est davantage centré car dans cette zone l'impact de l'injection par rapport à celui de l'éjection est relativement plus faible que dans la zone supérieure. La Figure 6 montre que toutes ces caractéristiques sont correctement représentées dans le modèle numérique. Cependant, le cœur d'air du vortex est légèrement plus large dans le modèle numérique que sur la photographie des expériences.

Les hauteurs d'eau mesurées par les capteurs à ultrason sont moyennées et comparées aux hauteurs d'eau moyennes issues du calcul numérique. La Figure 8 présente ces comparaisons. On remarque que les hauteurs issues du calcul numérique sont légèrement plus faibles que celles provenant des données expérimentales. Cependant ces différences sont inférieures à $5 \%$ et à l'incertitude des mesures sauf pour le capteur nommé C153. Ce dernier capteur présente des erreurs plus grandes que les autres pour toutes les configurations. La raison de ces différences n'est pas encore expliquée (problèmes dans la chaîne de mesure, défaut du modèle numérique pour cette position).

La Figure 9 compare des profils horizontaux de vitesse tangentielle issue des mesures par LDV et ceux provenant des calculs numériques. On note que les deux résultats sont proches sauf dans la région proche de l'interface au centre du tourbillon. Dans la zone proche du centre du tourbillon, le 


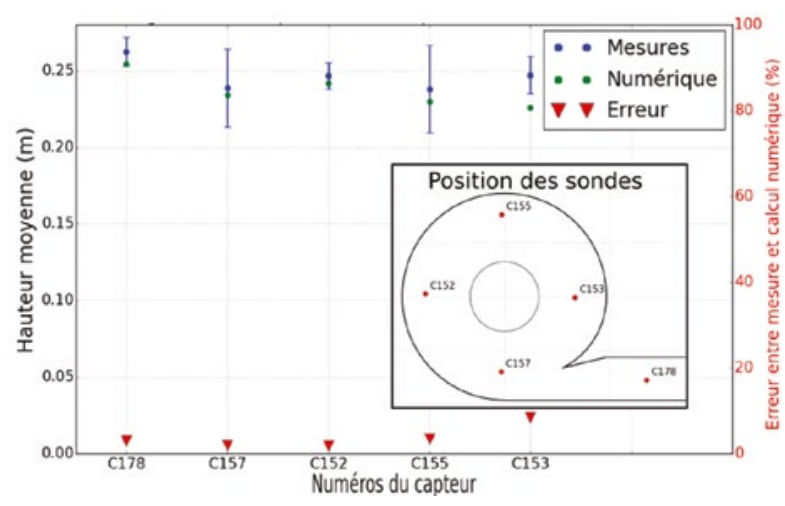

Figure 8 : Comparaison entre niveaux d'eau moyens mesurés et calculés pour la 5ième configuration. Les points verts et bleus sont respectivement les hauteurs d'eau moyennes calculées et mesurées. Les barres d'erreurs bleues sont les incertitudes de mesure. Les triangles rouges sont les écarts entre les deux niveaux d'eau. Ces derniers se rapportent à l'axe de droite. Les positions des capteurs sont rappelées dans la figure en cartouche.

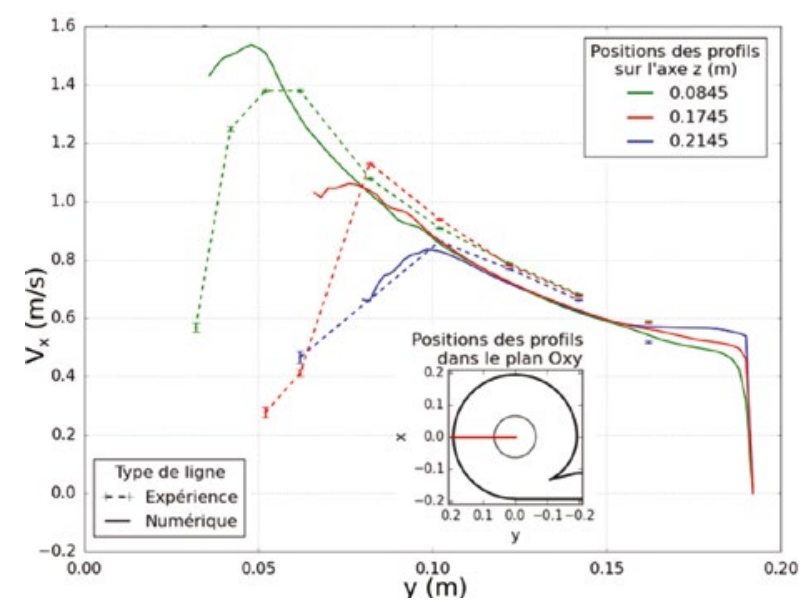

Figure 9 : Partie immergée de profils de vitesses tangentielles le long de lignes horizontales dans le plan $x=0$. Comparaison entre données numériques et expérimentales dans le cas de la configuration 5.

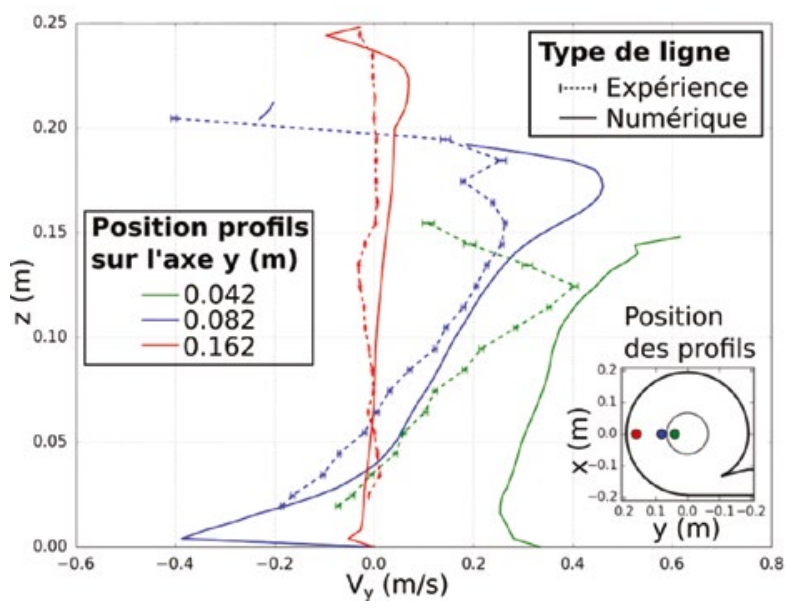

Figure 10 : Partie immergée de profils verticaux de vitesses radiales dans le plan $x=0$. Comparaison entre données numériques et expérimentales dans le cas de la configuration 5. modèle numérique surestime les vitesses tangentielles. Il faut remarquer que c'est dans cette zone que les incertitudes de mesures sont les plus grandes à cause des réflexions des lasers sur la surface libre. De plus, des vitesses sont mesurées à des endroits où il n'y a pas d'eau décelée dans le calcul numérique. Ceci confirme les analyses de la Figure 6, qui mettent en évidence que le tourbillon du modèle numérique est plus large (d'environ 10\%) que celui observé dans les expériences.

De la même manière, la Figure 10 montre des comparaisons entre modèle numérique et expériences pour les vitesses radiales. On constate que le modèle numérique représente correctement les vitesses radiales pour des points éloignés de plus de $4 \mathrm{~cm}$ de la surface libre (courbe rouge et le bas de la courbe bleu). Pour des points très proches de la surface libre les erreurs sont plus importantes comme pour les vitesses tangentielles. Dans ce cas les vitesses issues du calcul numérique sont plus grandes que les vitesses mesurées. Ces différences expliquent celles déjà observées entre les niveaux d'eau. En effet, les vitesses radiales sont liées au débit. Les vitesses radiales calculées étant plus importantes que celles mesurées, c'est le niveau d'eau qui s'adapte pour conserver le débit, d'où la présence d'un cœur du tourbillon plus large que dans la réalité.

\section{IV.2. Comparaison entre les différents modèles de turbulence}

Les comparaisons des niveaux d'eau mesurés et ceux obtenus avec le modèle k- $\varepsilon$ RNG montrent que ce dernier sous-estime la hauteur d'eau (écart de 8 à $30 \%$ pour les 5 sondes utilisées). Ceci est aussi observé sur la comparaison des profils immergés de vitesse (Figure 11). On constate par ailleurs que, dans la partie basse du profil, les vitesses déterminées par ce modèle présentent des écarts avec les données expérimentales plus importants que le modèle laminaire. Les écarts entre les vitesses tangentielles déterminées par ce modèle et celles mesurées sont de l'ordre de $35 \%$ dans la zone où le calcul laminaire modélise correctement les vitesses.

Les écarts entre les données expérimentales et les résultats du modèle k- $\omega$ SST sont beaucoup plus faibles. Ainsi, les hauteurs d'eau provenant du calcul avec le modèle k- $\omega$ SST sont légèrement plus élevées que celles extraites du calcul laminaire, elles présentent donc des écarts légèrement plus faibles avec les données expérimentales (ceci est retrouvé sur

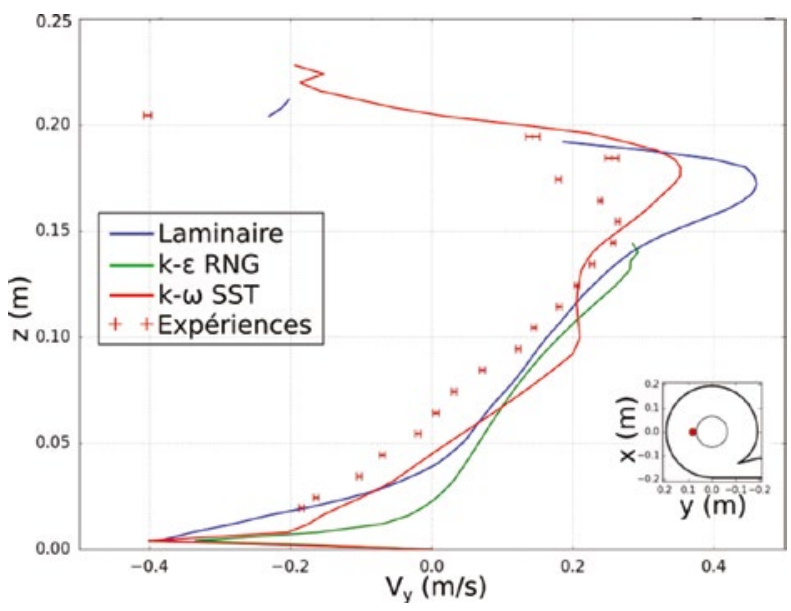

Figure 11 : Partie immergée de profils verticaux de vitesses radiales dans le plan $x=0$. Comparaison entre les données expérimentales, le calcul laminaire et les deux modèles de turbulence. 
la Figure 11). Les vitesses tangentielles sont très proches de celles obtenues avec le modèle laminaire (les écarts avec les données expérimentales sont inférieures à $5 \%$ dans la zone où le calcul laminaire modélise correctement les vitesses). Les vitesses radiales sont plus proches des données expérimentales que le modèle laminaire avec un pic de vitesse sous la surface libre un peu plus faible (Figure 11). Enfin proche $\mathrm{du}$ fond du bassin les vitesses verticales déterminées par le modèle k- $\omega$ SST sont significativement meilleures que celles déterminées par le modèle laminaire (écarts inférieurs à $15 \%$ en général et à $40 \%$ en certains points à comparer avec l'écart pour le modèle laminaire de $20 \%$ en général et $45 \%$ en certains points proches de la surface libre). Le modèle k- $\omega$ SST permet donc de représenter l'écoulement étudié un peu plus précisément que le modèle laminaire.

\section{CONCLUSIONS}

Un système expérimental permettant l'étude d'un tourbillon de vidange avec injection dans le but d'en extraire de l'énergie est présenté. Un modèle numérique du système sans extraction d'énergie est réalisé. On montre que les données issues du modèle numérique sont relativement proches des mesures réalisées, sauf pour les points les plus proches de l'interface air-eau. Le modèle de turbulence k- $\omega$ SST présente les résultats les plus proches des données expérimentales. Le calcul laminaire donne aussi de bons résultats. Pour modéliser l'extraction d'énergie, la turbine doit être ajoutée au modèle numérique. Pour cela, on pourra décrire l'écoulement autour de la turbine dans un repère tournant et celui proche du contenant dans un repère fixe (méthode Multiple Reference Frames, MRF). On pourra aussi utiliser les récents travaux de Vukčević et al. [2016] qui ont adapté la méthode de l'équilibrage harmonique (Harmonic Balance Method, HBM) à des écoulements diphasiques. Cette méthode se base sur les séries de Fourier des différentes variables et fait l'hypothèse que l'écoulement étudié est périodique et peut être représenté correctement par les premières harmoniques.

\section{REFERENCES}

Andersen A., T. Bohr B. Stenum J. J. Rasmussen, And B. Lautrup (2006) - The bathtub vortex in a rotating container. Journal of Fluid Mechanics, 556, 121, doi 10.1017/S0022112006009463.

Anwar H. O., J. A. Weller, AND M. B. Amphlett (1978) - Similarity Of Free-Vortex At Horizontal Intake. Journal of Hydraulic Research, 16(2), 95-105, doi 10.1080/00221687809499623.

Bøhling L., A. ANDERsen, AND D. FABRe (2010) - Structure of a steady drain-hole vortex in a viscous fluid. Journal of Fluid Mechanics, 656, 177-188, doi 10.1017/S0022112010001473.

Chassaing P. (2000) - Turbulence en mécanique des fluides : analyse du phénomène en vue de sa modélisation à l'usage de l'ingénieur, Cépaduès.

Chen Y., C. Wu M. Ye, AND X. Ju (2007) - Hydraulic characteristics of vertical vortex at hydraulic intakes. Journal of Hydrodynamics, Ser. B, 19(2), 143-149, doi 10.1016/ S1001-6058(07)60040-7.

Chen Y., C. Wu B. WANG, AND M. Du (2012) - Three-dimensional numerical simulation of vertical Vortex at Hydraulic intake. Procedia Engineering, 28, 55-60, doi 10.1016/ j.proeng.2012.01.682.
CRistofano L., AND M. NobiLi (2015) - Influence of boundary conditions in numerical simulation of free surface vortices. Energy Procedia, 82, 893-899, doi 10.1016/j.egypro.2015.11.836.

Cristofano L., M. Nobili, and G. Caruso (2014) - Experimental study on unstable free surface vortices and gas entrainment onset conditions. Experimental Thermal and Fluid Science, 52, 221-229, doi 10.1016/j.expthermflusci.2013.09.015.

Cristofano L., M. Nobili, and G. Caruso (2014) - Numerical evaluation of gas core length in free surface vortices. Journal of Physics: Conference Series, 547, 012030, doi 10.1088/1742 $-6596 / 547 / 1 / 012030$.

Cristofano L., M. Nobili G. P. Romano, and G. Caruso (2016) - Investigation on bathtub vortex flow field by Particle Image Velocimetry. Experimental Thermal and Fluid Science, 74, 130-142, doi 10.1016/j.expthermflusci.2015.12.005.

Daggett L. L., and G. H. Keulegan (1974) - Similitude in free-surface vortex formations. Journal of the Hydraulics Division, 100(11), 1565-1581.

Deshrande S. S., L. Anumolu, And M. F. Trujillo (2012) Evaluating the performance of the two-phase flow solver interFoam. Computational Science \& Discovery, 5(1), 014016, doi 10.1088/1749-4699/5/1/014016.

FOURESTIER G. (2017) - Modélisation expérimentale et numérique de l'écoulement au sein d'un système convertisseur de l'énergie de la houle, Université de Bretagne occidentale - Brest.

Hite JR., J., AND W. MiH (1995) - Velocity of air-core vortices at hydraulic intakes. Journal of Hydraulic Engineering, 121(8), 631-631, doi 10.1061/(ASCE)0733-9429(1995)121:8(631).

International Energy Agency (2015) - Key World Energy Statistics.

Li H., H. Chen Z. Ma, And Z. Yi (2008) - Experimental and numerical investigation of free surface vortex. Journal of Hydrodynamics, Ser. B, 20(4), 485-491, doi 10.1016/ S1001-6058(08)60084-0.

Lundgren T. S. (1985) - The vortical flow above the drain-hole in a rotating vessel. Journal of Fluid Mechanics, 155, 381-412, doi $10.1017 / \mathrm{S} 0022112085001860$.

OdgAARD A. (1986) - Free-surface air core vortex. Journal of Hydraulic Engineering, 112(7), 610-620, doi 10.1061/(ASCE) 0733-9429(1986)112:7(610).

OPENFOAM FOUNDATION (2013) - OpenFOAM - User Guide.

Power C., A. Mcnabola, And P. Coughlan (2016) - A parametric experimental investigation of the operating conditions of gravitational vortex hydropower (GVHP). Journal of Clean Energy Technologies, 4(2), 112-119, doi 10.7763/JOCET.2016.V4.263.

Rankine W. J. M. (1872) - A Manual of Applied Mechanics, Charles Griffin.

ReCoquillon Y. (2013) - Etude expérimentale et numérique des écoulements diphasiques dans la boîte à eau d'un véhicule automobile. Ph.D. dissertation, Université d'Orléans, Orléans, France.

Speziale C. G., AND S. Thangam (1992) - Analysis of an RNG based turbulence model for separated flows. International Journal of Engineering Science, 30(10)1379-IN4, doi 10.1016 /0020-7225(92)90148-A.

STEPANYANTS Y., AND G. YeOH (2008) - Stationary bathtub vortices and a critical regime of liquid discharge. Journal of Fluid Mechanics, 604(1), 77-98.

VukčEvić V., G. CVijetić I. GATin, AND H. JASAK (2016) - The Harmonic balance method for temporally periodic free surface flows in marine hydrodynamics, Guimaraes, Portugal.

ZOTLÖTERER F. (2004) - Hydroelectric power plant. 